\title{
An Approach for Detecting Polarity of Online Product Reviews Based on Opinion Target and Opinion Words from Reviews
}

\author{
${ }^{1,2}$ Shri Ramdeobaba College of Engineering and Management, Nagpur, Maharashtra, India. \\ Email: bhongadebg@rknec.edu,zadgaonkarav1@rknec.edu
}

\section{Received: 09 ${ }^{\text {th }}$ July 2018, Accepted: $14^{\text {th }}$ August 2018, Published: $31^{\text {st }}$ August 2018}

\begin{abstract}
Online shopping is a medium for many consumers to buy goods and services from E-commerce applications. The public is more inclined to online shopping from the last decade. These online shopping portals also provide the customers a way to express their experience with the product by giving reviews. These reviews not only prove a benefit to the consumer but also help the manufacturer in improving the quality of their products. Our proposed approach works on determining the polarity of the mobile phone's reviews i.e. positive, negative or neutral using natural language processing techniques. We hope that our proposed model will act as a useful tool for online product review mining.
\end{abstract}

Keywords: Opinion Target, Opinion Word, SemiSupervised Method, Online Product Reviews, Semantic Analysis.

\section{Introduction}

Nowadays online shopping has become a new trend of shopping. Myriad of people are attracted to this trending way of shopping. Online shopping portals avail the consumer of expressing their opinion about a product using reviews. These reviews not only help the general public in gaining the knowledge of the product but also the manufacturer to improve the quality of the product. Our proposed approach works on determining the polarity online product review using natural language processing tools.

For example the review "The camera is awesome but the battery life is poor" here the reviewer has expressed positive opinion towards the camera but negative opinion towards battery-life. Our system works on determining fine grain polarity of the review. To achieve this, firstly we need to extract opinion target and opinion words from the review. The opinion target is the words about which an opinion is given. Like in this review words like "battery" and "camera" are opinion target. And opinion words are the words that express the opinion about the product. In the given example opinion words are "awesome" and "poor" our main aim is to determine the relation between the opinion targets and opinion words. Our proposed system has collected mobile review dataset from Amazon website. After that, we have identified opinion target and opinion words using rule-based pattern. Opinion words are assigned some weight. Some threshold value was decided, based on which the classification of the polarity was performed.

\section{Related work}

There are mainly two methods used for sentiment analysis using the classification namely supervised method and unsupervised method. The supervised methods have some prior knowledge that can be useful for classification of the text using machine-learning algorithms like naive Bayes, SVM and maximum entropy. The unsupervised method does not have prior knowledge of the classification. Hence unsupervised method needs to discover certain syntactic patterns that are useful to express opinions.

The author Subhabrata Mukherjee, Pushpak Bhattacharyya, [13], [3], [6], and [15] used a novel method to figure out expressions of opinion in product reviews with different sentiment and features. They used Direct Neighbour Relation and Dependency Relation for Relation extraction to classify the extracted opinion words as positive or negative using Rule-Based Classification and Supervised Classification. The author Amani K Samha P. P. [2], [4] have focused on aspect extraction at a sentence level using different NLP techniques. The first task is to prepare the dataset by employing NLP techniques. The second task is to find Opinion words and map them to the product aspect. Supervised Methods for AspectBased Sentiment Analysis is used in [5], [11], [12] by author Hussam Hamdan, Patrice Bellot, Frederic Béchet. The author Nishantha Medagoda, S. S have focused on our contribution in SemEval2014. The author used CRF model with different features for aspect extraction. Z-score model for categorydetection Multinomial Naive Bayes for polarity detection. In the [14], [8], [9], [7] paper, the author Toqir A. Rana, Yu$\mathrm{N}$ Cheah, has used a two-fold rule-based method. In the first fold, SPRs are been used for the extraction of nouns/noun phrases as aspects and associated opinions using opinion lexicon. In the second fold, the proposed model searches aspects associated.

\section{Dataset and Methods}

In the proposed system online mobile phone's reviews are taken from JSON file of Cell Phones and Accessories [21]. This dataset contains following information for sentiment analysis. 
Reviewer ID - It is the identity of reviewer to separate from one another.

As in - It is the identity if the product.

Helpful - It represent how much helpful was the rating of a review for the product.

Overall - It represent the overall rating reviewer had given to the product.

Reviewer Name - It represent the name of the reviewer.

Review Text - It is the original text of reviewer which was used for classification.

Summary - It represent the summary of the review Text.

Unix Time - It represent the unix time when review was taken.

Review Time - It represents the raw time in the format of DD MM, YYYY when the review was taken.

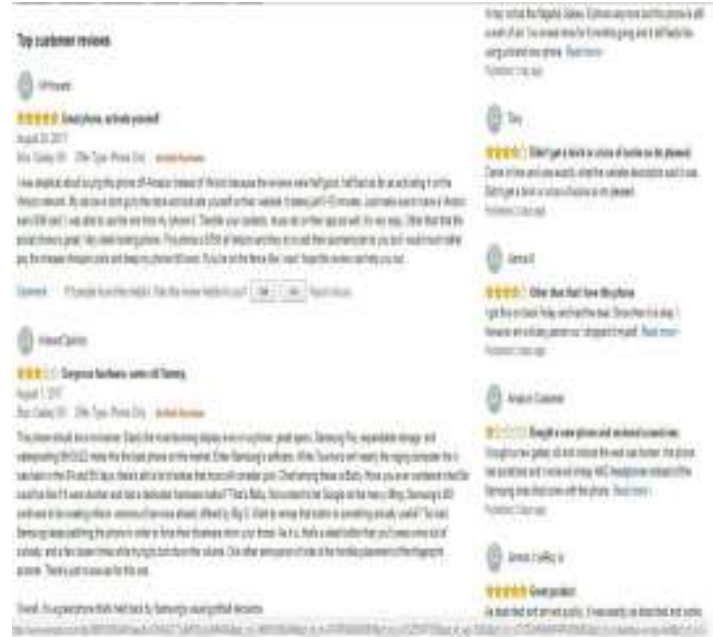

Figure 1. Screenshot of Mobile Phone Review taken from Amazon.com.

On this dataset further pre-processing like stop word removal is carried out, to remove the frequently occurring words like a, the, is, it, on etc which contribute little to the uniqueness of the sentence. Preprocessing also includes correction of words and removing unwanted symbols. Given below is the general architecture of the proposed system.

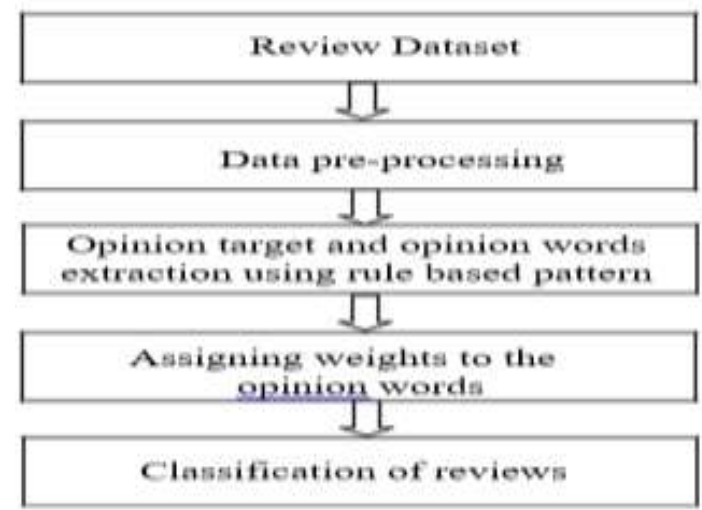

Figure 2. Architecture of the Proposed System.
In the classification of reviews, we classify the reviewed text as positive, negative or neutral by assigning weight to each opinion words and adding them. The opinion decision was done after normalization of the final score across all reviewed text. Following are the steps of the proposed algorithm derived for the architecture of the proposed system:

\section{Proposed Algorithm}

Step 1: Reviews are collected about the mobile phone from the Amazon site.

Step 2: Pre-processing like stop-word removal is performed on this reviews. Hence, the commonly occurring words are removed.

Step 3: Most frequent features of mobile like "Screen", "display", "battery", "sim-card", “application", "software" are selected man ally. These are called the target words about which mostly an opinion is given.

Step 4: After collecting the target words the next the step is to collect all the opinion words related to this target words. Some rule-based method is used for extracting opinion target and opinion words from the review [1]. These rules are generated from patterns. We used eight patterns to extract opinion target and its related opinion words using part of speech tagging [1]. These patterns are listed in table 1.

Step 5: The last step is to perform the classification using weights assigned to opinion words. The weights below 0.2 are for negative polarity, weights between 0.2 and 0.8 are for neutral polarity and above 0.8 are positive polarity. Some rule-based method is used for extracting opinion target and opinion words from the review [1].

\begin{tabular}{|c|c|c|c|}
\hline Pattons & $\begin{array}{l}\text { Finet } \\
\text { varouses }\end{array}$ & $\begin{array}{l}\text { Neset } \\
\text { wored }\end{array}$ & $\begin{array}{l}\text { Nert } \\
\text { wores }\end{array}$ \\
\hline Tattera 1 & Is & MTRESAMT & - \\
\hline Pattern ? & $x y$ & NTNERNN & $\begin{array}{l}\text { NivE } \\
\text { NTS }\end{array}$ \\
\hline Pattern 3 & $\begin{array}{l}\text { REBPERS } \\
\text { hen }\end{array}$ & 33 & - \\
\hline Pattern 4 & $\begin{array}{l}\text { IEDINARDS } \\
\text { TRE }\end{array}$ & 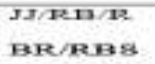 & $\begin{array}{l}\text { NANE } \\
\text { NMN }\end{array}$ \\
\hline Pattura 3 & $\begin{array}{l}\text { RERTRAS } \\
\text { TEB }\end{array}$ & $\begin{array}{l}\text { VBD } \\
\text { VAR }\end{array}$ & - \\
\hline Pattera 6 & $\begin{array}{l}\text { RAPRAS } \\
\text { / REB }\end{array}$ & $\begin{array}{l}\text { RAR } \\
\text { FEBS, FEB }\end{array}$ & y \\
\hline vattara 7 & $\begin{array}{l}\text { VBD } \\
\text { Van }\end{array}$ & MTNEANN & $=$ \\
\hline Pattora : & $\begin{array}{l}\text { vabi } \\
\text { vave }\end{array}$ & 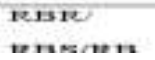 & - \\
\hline
\end{tabular}

Table 1. Eight Pattern Used for Extracting Opinion Target and Words.

NLP Techniques for Text Preprocessing Opinion mining requires various reprocessing steps for extracting features and structure of the text. These preprocessed steps include word segmentation, tokenization, Part of Speech (POS) tagging, parsing, spelling correction, etc. We use following toolkits and libraries for NLP in Python. 


\begin{tabular}{|l|l|}
\hline Loolkits/ & \multicolumn{1}{|c|}{ Description } \\
\hline NLTK & $\begin{array}{l}\text { Natural Language Toolkit } \\
\text { (NLTK). This library preforms } \\
\text { Natural Language Processing } \\
\text { tasks like POS tagging, } \\
\text { stemming, parsing, semantic } \\
\text { reasoning, tokenization, etc }\end{array}$ \\
\hline WordNet & $\begin{array}{l}\text { NLTK also provides many } \\
\text { corpora interface and lexicons } \\
\text { for semantic analysis and } \\
\text { opinion mining. WordNet is the } \\
\text { NLTK corpus reader and lexical } \\
\text { database for English words. It } \\
\text { creates synsets by grouping } \\
\text { English words into synonyms } \\
\text { sets. }\end{array}$ \\
\hline TextBlob & $\begin{array}{l}\text { TextBlob is a Python library. It } \\
\text { is created for textual data } \\
\text { processing. It has simple API } \\
\text { for tasks like part-of-speech } \\
\text { tagging, } \\
\text { classification, translation, } \\
\text { analysis, noun phrase extraction } \\
\text { and many more }\end{array}$ \\
\hline
\end{tabular}

Table 2. Toolkits and Libraries used along with their Description.

Words are extracted as opinion words from opinion target by analysing behaviour pattern from Table 1 . These extracted words only represent the opinion of the reviewer [15]. For example, in pattern1, pattern2 $\&$ pattern 4 mentioned in the table, NN/NNS represent nouns in the review sentence. Therefore, it will act as an opinion target and corresponding $\mathrm{JJ}$ act as an adjective, which represents opinion word of opinion target NN/NNS.

After interpreting opinion words correctly we can calculate the corresponding score of each opinion words using sentiment property of TextBlob library. The sentiment property returns a tuple Sentiment (polarity, subjectivity). The polarity in the tuple represents the score containing float value. The polarity float value ranges from -1.0 to 1.0 . The subjectivity in the tuple represents the float value ranging from 0.0 to 1.0 . The subjectivity score of 0.0 represents that the subject was very objective and the subjectivity score of 1.0 represents that the subject is very subjective. The score is calculated in this way from above mentioned extracted opinion words. The final score for each review is calculated by adding sentiment polarity score generated for extracted opinion words in that review text. This score act as a final weight which is to identify the polarity of the review text.

\section{Results and Discussion}

In this paper, we have collected the reviews from Amazon website in the JSON format. We have used NLP techniques to extract opinion target and opinion words from the reviews. Then we used WorldNet to find the synonyms for each extracted opinion word. This forms the local corpus of the opinion words. The opinion words which are present in the local corpus are checked in each reviewed text and corresponding opinion words are extracted to calculate the Sentiment (polarity, subjectivity) score from TextBlob library in Python for every extracted word. The Sentiment (polarity, subjectivity) score for every extracted word for every reviewed text was added together to generate final reviewed text score. This final score for each reviewed text then acts as a weight. Based on these weight assigned to the every reviewed text, the classification of the polarity was done. The weights were normalized before performing classification. The final weights below 0.2 are used for negative polarity. Final weights within the range of 0.2 to 0.8 are used for neutral polarity and the final weight above 0.8 are used positive polarity.

Following are some sample examples of review dataset with confusion matrix of the result of the testing dataset.

\section{Example 1:}

Review Text: "Yo get exactly what you order in a timely fashion. And the item is just as described. Great buy if you ask me."

Opinion target: \{'buy', 'buy ask', 'fashion'\}

Opinion word: ['great', 'timely']

Score of opinion words: $[0.8,0.0]$

Sum of score: [0.8]

Classification: Positive.

\section{Example 2:}

Review Text: "I bought this for the at\&t samsung galaxy s2 and the seller was veryery slow in getting this item to me.

I also notice that this product do fit the SGS2, but the cut outs was NOT good. The cut out opening was too small for the power button, there was also an extra cut out for no reason. It does fit nice so I gave it 2 stars, but bad opening for my phone will be costly."

Opinion target: \{'power', 'power button', 'product', 'product fit', 'samson', 'stars'\}

Opinion word: ['NOT good', 'nice', 'galaxy', 'slow', 'small', 'bad']

Score of opinion words: $[-0.35,0.0,-0.25,-0.7]$

Sum of score: [-1.6]

Classification: Negative. 


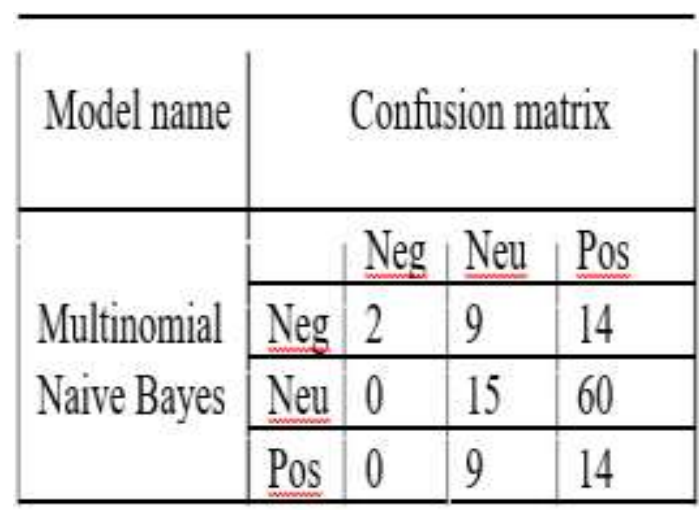

Table 3. Confusion Matrices of Five Different Models.

\begin{tabular}{|c|c|c|c|c|}
\hline & \multicolumn{2}{|r|}{$\mathrm{Neg}$} & \multirow{2}{*}{ Neu } & \multirow{2}{*}{$\frac{\text { Pos }}{15}$} \\
\hline & $\mathrm{Neg}$ & 3 & & \\
\hline \multirow{3}{*}{ Naive Bayes } & $\mathrm{Neu}$ & 9 & 19 & 47 \\
\hline & & & & \\
\hline & Pos & 9 & 30 & 111 \\
\hline \multirow{4}{*}{$\begin{array}{l}\text { Logistic } \\
\text { Regression }\end{array}$} & & Neg & Neu & Pos \\
\hline & Neg & 3 & 20 & 2 \\
\hline & Neu & 4 & 47 & 24 \\
\hline & Pos & 5 & 36 & 109 \\
\hline \multirow{4}{*}{ Linear SVM } & & $\mathrm{Neg}$ & Neu & Pos \\
\hline & Neg & 5 & 17 & 3 \\
\hline & Neu & 5 & 44 & 26 \\
\hline & Pos & 5 & 34 & 111 \\
\hline \multirow{4}{*}{$\begin{array}{l}\text { Random } \\
\text { Forest } \\
\text { Classification }\end{array}$} & & $\mathrm{Neg}$ & Neu & Pos \\
\hline & $\mathrm{Neg}$ & 0 & 20 & 5 \\
\hline & Neu & 2 & 23 & 20 \\
\hline & Pos & 0 & 37 & 113 \\
\hline
\end{tabular}

We have performed classification on the polarity of review using assigned weights of opinion words for each review text. The $25 \%$ review text was used for testing the machine learning classification model after training $75 \%$ review text with its polarity.

Linear SVM solves the problem that is linearly separable. If linear isn't working for the above problem then the next step is to use non-linear SVM which typically tries to figure out the correlation in higher dimensional feature space. Our result shows non-linear kernel (Gaussian, polynomial and Sigmoid) in SVM doesn't significantly improve models performance when compared with linear SVM. Decision tree breaks down the dataset into smaller and smaller subsets with simultaneously building associated decision structure of underline text review. Decision tree classifier possesses the series of carefully crafted questions until a conclusion about a class labelled record is reached. Random forest builds multiple decision trees and merges them together to get the more stable classification. Our result shows that random forest increases accuracy for decision tree classifier. Multinomial Naïve Bayes estimates the conditional probability of a particular word given a class. This probability comes from relative frequency of that word in the review text belonging to the class. It also takes into account multiple occurrences of that word. Our result shows that Multinomial Naïve Bayes performed better than non-linear kernel in SVM.
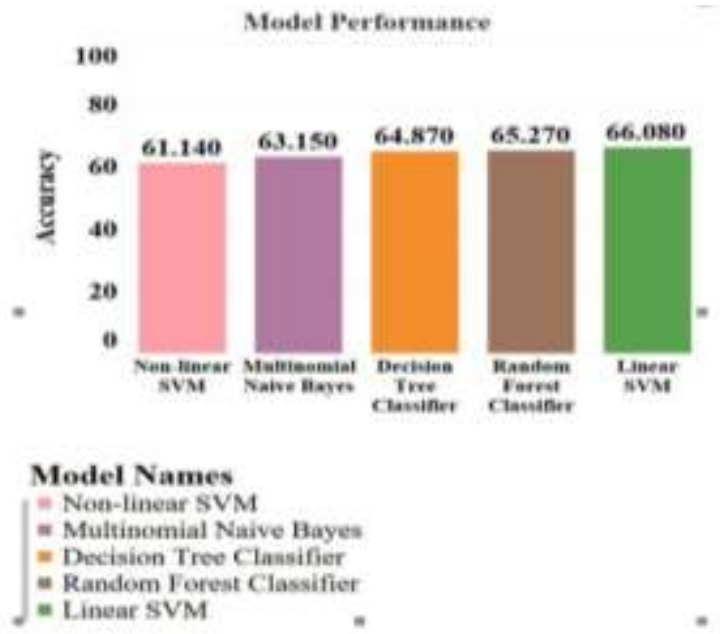

Figure 3. Model Performance of Proposed System.

\section{Conclusion}

The proposed approach works on the classification of reviews of mobile phone taken from Amazon.com. We have used NLP techniques for extraction of opinion target and opinion words. We have tested the accuracy of different classifiers for the classification of the reviews. Figure 3 shows a graphical representation of the accuracy obtained from five different models. The accuracy obtained from Linear SVM is the highest i.e. 66.08. The second high accuracy is for Random Forest Classification i.e. 65.27. In the future, we would like to extend this work on some other product review and try to increase the accuracy of the proposed model.

\section{References}

[1] Htay, s. S., \& lynn, k. T. (2013). Extracting product features and opinion words using pattern knowledge in customer reviews. The scientific world journal, 2013.

[2] Anisha P Rodrigues, D. N. (2016). "Mining Online Product Reviews And Extracting Product Features Using Unsupervised Method". IEEE.

[3] Balakrishnan Gokulakrishnan, P. P. (2012). "Opinion mining And Sentiment Analysis On A Twitter Data Stream". The International Conference On Advances In Ict For Emerging Region, IEEE.

[4] Sentiment Analysis". International Workshop On Semantic Evaluation,Dublin, Ireland. 
[5] Cho, M. Y.-P. (May 31 - June 5, 2015). "Lcct: A Semi-Supervised Model For Sentiment Classification". Association For Computational Linguistics.

[6] Hussam Hamdan, P. B. (August 23-24 2014). Supervised Methods For Aspect-Based Sentiment Analysis". International Workshop On Semantic Evaluation,Dublin, Ireland

[7] Kang Liu, L. X. (March 2015). "CoExtracting Opinion Targets And Opinion Words From Online Reviews Based On The Word Alignment Models". Ieee Transactions On Knowledge And Data Engineering, Vol. 27, No.3.

[8] Toqir A. Rana, Y.-N. C. (2017. ). "A TwoFold Rule-Based Model For Aspect Extraction". Universiti Sains, Malaysia.

[9] Samha, A. K. (Jan - Feb 2016). "AspectBased Opinion Mining Using Dependency Relations" . International Journal Of Computer Science Trends And Technology (Ijcst) - Volume 4 Issue 1.

[10] Subhabrata Mukherjee, P. B. (2012). "Feature Specific Sentiment Analysis For Product Reviews". Computational Linguistics And Intelligent Text Processing , 475-487.

[11] Bhagyashree G. Bhongade, \& Ashwini V. Zadgaonkar. (2018). A Semi-supervised approach for Opinion Mining Using Online Product Reviews. Accepted in International Conference on Recent Trends in Engineering \& Science (ICRTES). To be published in coming issues Vol.8 of International Journal of Engineering and Technology (UAE). EISSN: 2227-524X.

[12] Ashwini Zadgaonkar, Rasika Landge, “ Facet extraction from queries from search results", International journal of Engineering and Applied Sciences 13, (3) 575-579, $2018 @$ Medwell Journals , 2018.

[13] Ashwini A. Shende, Avinash J. Agrawal\& Dr. O. G. Kakde, "Domain Specific Named Entity Recognition Using Supervised Approach ", International Journal of Computational Linguistics (IJCL), Volume (3) : Issue (1) : 2012, pp. 66-78.

[14] A. V. Zadgaonkar, A. J. Agrawal, S. Aote, "Facets extraction-based approach for query recommendation using data mining approach", International Journal of Engineering \& Technology, 7 (1) (2018) 121-125, pp.121-125.

[15] Ashwini V. Zadgaonkar, "Natural Language Understanding Using Open Information Extraction Technique", International Journal of Computer Sciences and Engineering, Vol.6, Issue.1, pp.347-350, 2018.

[16] http://jmcauley.ucsd.edu/data/amazon/ 